\title{
Influences of blood lipids on the occurrence and prognosis of hemorrhagic transformation after acute cerebral infarction: a case-control study of 732 patients
}

Gang LV' ${ }^{1}$ Guo-qiang Wang ${ }^{2 *}$, Zhen-xi Xia², Hai-xia Wang ${ }^{2}$, Nan Liu², Wei Wei², Yong-hua Huang ${ }^{2}$ and Wei-wei Zhang ${ }^{2}$

\begin{abstract}
Background: To study the influence of blood lipid levels on hemorrhagic transformation $(\mathrm{HT})$ and prognosis after acute cerebral infarction (ACl).

Methods: Patients with ACl within 72 h of symptoms onset between January 1st, 2015, and December 31st, 2016, were retrospectively analyzed. Patients were divided into group A (without HT) and group B (HT). The outcomes were assessed after 3 months of disease onset using the modified Rankin Scale (mRS). An mRS score of 0-2 points indicated excellent prognosis, and an mRS score of 3-6 points indicated poor prognosis.
\end{abstract}

Results: A total of 732 patients conformed to the inclusion criteria, including 628 in group A and 104 in group B. The incidence of HT was 14.2\%, and the median onset time was $2 \mathrm{~d}$ (interquartile range, 1-7 d). The percentages of patients with large infarct size and cortex involvement in group B were 80.8 and $79.8 \%$, respectively, which were both significantly higher than those in group $A$ ( 28.7 and $33.4 \%$, respectively). The incidence rate of atrial fibrillation (AF) in group B was significantly higher than that in group A (39.4\% vs. $13.9 \%, P<0.001)$. The adjusted multivariate analysis results showed that large infarct size, cortex involvement and AF were independent risk factors of $\mathrm{HT}$, while total cholesterol (TC) was a protective factor of $\mathrm{HT}(\mathrm{OR}=0.359,95 \% \mathrm{Cl} 0.136-0.944, P=0.038)$. With every $1 \mathrm{mmol} / \mathrm{L}$ reduction in normal TC levels, the risk of $\mathrm{HT}$ increased by $64.1 \%$. The mortality and morbidity at 3 months in group B (21.2 and 76.7\%, respectively) were both significantly higher than those in group A ( 8.0 and $42.8 \%$, respectively). The adjusted multivariate analysis results showed that large infarct size $(O R=12.178,95 \% \mathrm{Cl} 5.390-27.516, P<0.001)$ was an independent risk factor of long-term unfavorable outcomes, whereas low-density lipoprotein cholesterol $(L D L-C)$ was a protective factor $(O R=0.538,95 \% \mathrm{Cl} 0.300-0.964, P=0.037)$. With every $1 \mathrm{mmol} / \mathrm{L}$ reduction in normal LDL-C levels, the risk of an unfavorable outcome increased by $46.2 \%$. Major therapies, including intravenous recombinant human tissue plasminogen activator (rTPA), intensive lipid-lowering statins and anti-platelets, were not significantly related to either HT or long-term, post-ACl poor prognosis.

(Continued on next page)

\footnotetext{
*Correspondence: wgqbj@163.com

²Department of Neurology, Chinese PLA Army General Hospital, Beijing

100700, China

Full list of author information is available at the end of the article
}

(c) The Author(s). 2019 Open Access This article is distributed under the terms of the Creative Commons Attribution 4.0 International License (http://creativecommons.org/licenses/by/4.0/), which permits unrestricted use, distribution, and reproduction in any medium, provided you give appropriate credit to the original author(s) and the source, provide a link to the Creative Commons license, and indicate if changes were made. The Creative Commons Public Domain Dedication waiver (http://creativecommons.org/publicdomain/zero/1.0/) applies to the data made available in this article, unless otherwise stated. 
(Continued from previous page)

Conclusion: For patients with large infarct sizes, especially those with cortex involvement, AF, or lower levels of TC, the risk of $\mathrm{HT}$ might increase after $\mathrm{ACl}$. The risk of a long-term unfavorable outcome in these patients might increase with a reduction in LDL-C.

Keywords: Acute cerebral infarction, Hemorrhagic transformation, Total cholesterol, Low-density lipoprotein, Intensive lipid-lowering statins, Anti-platelet, Atrial fibrillation; modified Rankin scale

\section{Background}

Hemorrhage occurring in the infarction site during acute cerebral infarction (ACI) is called hemorrhagic transformation (HT) [1] and is a complication of ACI. According to the degree of hemorrhage, HT is divided into punctate and patchy hemorrhagic infarction (HI) and space-occupying parenchymal hematoma $(\mathrm{PH})$. $\mathrm{HI}$ is further divided into the HI-1 type (small petechiae) and the HI-2 type (more confluent petechiae, without a space-occupying effect), and $\mathrm{PH}$ is further divided into the $\mathrm{PH}-1$ type (hematoma < $30 \%$ infarct size, mildly space-occupying) and the $\mathrm{PH}-2$ type (hematoma $>30 \%$ infarct size, significantly space-occupying) [2]. The incidence of HT, its influences on prognosis, and the risk factors of its formation remain unclear. This study aimed to evaluate the incidence of HT, long-term unfavorable outcome at 3 months after HT, and the risk of HT development to investigate the influence of blood lipids on HT and prognosis after ACI.

\section{Material and methods Study subjects}

ACI patients who were continuously admitted and treated in the Department of Neurology of Chinese PLA Army General Hospital between January 1st, 2015 and December 31st, 2016, were retrospectively analyzed. This study was approved by the Medical Ethics Committee of the Chinese PLA Army General Hospital.

\section{Inclusion criteria}

1) Patients who conformed to the diagnostic criteria of ACI [3] and 2) patients with $\leq 72 \mathrm{~h}$ between disease onset and hospital admission.

\section{Exclusion criteria}

1) Patients who had a past history of nervous system impairment caused by stroke and a modified Rankin Scale score $(\mathrm{mRS}) \geq 2$ points; and 2) patients who had combined liver or kidney damage, blood system diseases, coagulation dysfunction, connective tissue diseases, thyroid dysfunction, tumors, cerebral aneurysm, and cerebrovascular malformation.

\section{Grouping}

Patients were divided into a without HT (A) group and an HT (B) group. Due to limitations in sample size, all types of HT (HI types 1 and 2 and PH types 1 and 2) were included in group $B$.

\section{Definition of major indicators}

Based on brain magnetic resonance imaging (MRI) or computed tomography (CT) images, the infarct sizes were divided into: 1 ) large infarct size (infarct lesion $>3 \mathrm{~cm}^{2}$ with 2 or more vascular blood supply areas involved); 2) medium infarct size (infarct lesion $1.5 \sim 3.0 \mathrm{~cm}^{2}$ with 1 vascular branch blood supply area involved); and 3) small (lacunar) infarct size (infarct lesion $<1.5 \mathrm{~cm}^{2}$ ) [4]. HT was confirmed and categorized according to the appearance of abnormally high density on repeated CT films, or hypointensity signals on SWI or GRE sequences of MRI. The second CT was routinely performed within $24 \mathrm{~h}$ for patients who received intravenous recombinant human tissue plasminogen activator (rTPA), even if their conditions were stable; for others, the second CT was performed at any time if their symptoms or signs were aggravated. If a patient's condition was continuously stable, a follow-up CT was ordinarily performed before discharge. If permitted, MRI with SWI or GRE sequences for all patients were performed at the appointed time, which was limited by the number of waiting queues and generally took approximately one week.

Sustained or paroxysmal atrial fibrillation (AF) was confirmed by an urgent ECG when a patient arrived at the emergency department; then, a routine ECG and past history provided by the patient or their relatives were obtained after admission. Further, long-term ECG monitoring was performed for all patients at least in the initial several days until the condition stabilized. A history of hyperlipidemia referred to past hyperlipidemia and continuous use of statins. Blood pressure was measured when the patient was sent to the emergency room or admitted into the hospital. The blood glucose was the random blood glucose result when the patient was sent to the emergency room or admitted into the hospital. The blood lipid and plasma protein levels were the fasting test results in the morning after the patient was admitted into the hospital. Hypoproteinemia (used to evaluate whether a patient was frail or malnourished) referred to both a total protein level lower than $60 \mathrm{~g} / \mathrm{L}$ and an albumin level lower than $35 \mathrm{~g} / \mathrm{L}$. The thrombolysis dose of intravenous recombinant tissue-type plasminogen activator (rTPA) was $0.9 \mathrm{mg} / \mathrm{kg}$. The double anti-platelet therapy consisted 
of oral administration of aspirin $\geq 100 \mathrm{mg}+$ clopidogrel hydrogen sulfate tablet $75 \mathrm{mg} / \mathrm{d}$. The intensive lipidlowering statin (SILL) treatment consisted of an oral administration of atorvastatin $\geq 40 \mathrm{mg} / \mathrm{d}$.

\section{Outcome}

The outcome after 3 months of ACI was assessed using the mRS: $0=$ no symptoms; $1=$ symptoms without significant dysfunction; 2 = slight disability and inability to perform all previous activities with ability to look after daily affairs without assistance; $3=$ moderate disability requiring some help with ability to walk without assistance; $4=$ moderately severe disability and inability to walk or attend to bodily needs without assistance; $5=$ severe disability, bedridden, incontinent, and requiring constant care and attention; and $6=$ death. Follow-up was completed by physicians in the Department of Neurology whom did not participate in the treatment and was conducted at patients' subsequent visits or by telephone communication.

\section{Statistical analyses}

Data were analyzed using PASW Statistics (formerly SPSS Statistics) (๑Copyright IBM Corporation 2016, IBM Corporation Software Group Route 100 Somers, NY 10589, USA). Quantitative data conforming to a normal distribution are expressed as $X \pm s$. Quantitative data that did not conform to a normal distribution are expressed using $\mathrm{M}\left(\mathrm{Q}_{1}-\mathrm{Q}_{3}\right)$. Baseline quantitative data conforming to a normal distribution and homogeneity of variance were examined using the analysis of variance (ANOVA); otherwise, the Wilcoxon rank sum test was performed. The comparison of baseline qualitative data between groups was performed using the $\chi^{2}$ test or the rank sum test. For analyses of the risk factors of HT and the factors influencing poor $\mathrm{ACI}$ prognosis, the raw data were converted into categorical variables (multicategory variables were grouped based on dummy variables) for nonconditional binary logistic regression analyses using the ENTER method. $P<0.05$ indicated that the difference had statistical significance.

\section{Results}

\section{Data sets assessed during the study interval}

A total of $811 \mathrm{ACI}$ patients were continuously enrolled over 2 years. After enrollment, 76 patients who did not conform to the criteria were excluded (combined with tumors, liver or kidney insufficiency, intracranial infection, intracranial aneurysm, blood system diseases, hypothyroidism, and loss of follow-up at 3 months). A total of 732 patients were enrolled, including 628 in group A (no-HT) and 104 in group B (HT, Fig. 1).

\section{Baseline characteristics}

The age, gender, history of hypertension, history of diabetes mellitus and hyperlipidemia, and blood pressure at admission between these two groups were not significantly different. The overall incidence of AF was $17.5 \%$, and the incidence of AF in group A was significantly lower than that in group B $(13.9 \%$ vs. $39.4 \%, P<0.001)$. The blood glucose in group $B$ was significantly higher than that in group A. TC and TG in group B were significantly lower than those in group $\mathrm{A}$. The percentage of cases with low LDL-C in group B was significantly higher than that in group A. High-density lipoprotein cholesterol (HDL-C) levels and hypoproteinemia were not significantly different between these two groups (Table 1).

\section{Site and size of $\mathrm{ACl}$}

More than 2/3 of infarction sites were distributed in the anterior circulation. The percentages of the large, medium, and small infarct sizes were $15.2 \%, 20.9 \%$, and $63.9 \%$, respectively. The incidence of HT was $14.2 \%$ (104/732). The onset time was $0-26 \mathrm{~d}$, with a mean of $2 \mathrm{~d}(1-7 \mathrm{~d})$. The percentages of HT corresponding to large, middle, and small infarct sizes were $37.8 \%$ (42/111), 27.5\% (42/153), and $4.3 \%$ (20/468), respectively. HT incidence in anterior circulation infarctions was significantly higher than that in vertebrobasilar artery system infarctions $(89 / 526=16.9 \%$ vs. $15 / 206=7.3 \%$ ). The percentages of large and medium infarct sizes and cortex involvement in group B were $80.8 \%$ and $79.8 \%$, respectively, which were both significantly higher than the corresponding percentages of $28.7 \%$ and $33.4 \%$ in group A (Table 2).

The percentages of large, medium, and small ACI sizes in $\mathrm{AF}$ patents were $37.5 \%, 34.4 \%$, and $28.1 \%$, respectively, and the corresponding HT incidence rates were $56.1 \%$, $36.6 \%$, and $7.3 \%$, respectively (Table 3 ). The differences between group A and group B were not significant (Table 3).

\section{AF-associated $\mathrm{ACl}$}

Among patients with AF-associated ACT, the percentage of HT with cortex involvement (40.7\%) was significantly higher than that without cortex involvement (14.3\%). Among patients with sinus arrhythmia-associated ACI, the percentage of HT with cortex involvement (23.2\%) was also significantly higher than that without cortex involvement (3.8\%) (Table 4).

National Institutes of Health stroke scale (NIHSS) estimation Statistical analyses based on the stratification of the degree of the baseline NIHSS showed that HT in patients with NIHSS $>4$ points in group B was significantly higher than that in group A $(76.0 \%$ vs. $46.3 \%$, $P<0.001$ ) (Table 5). 


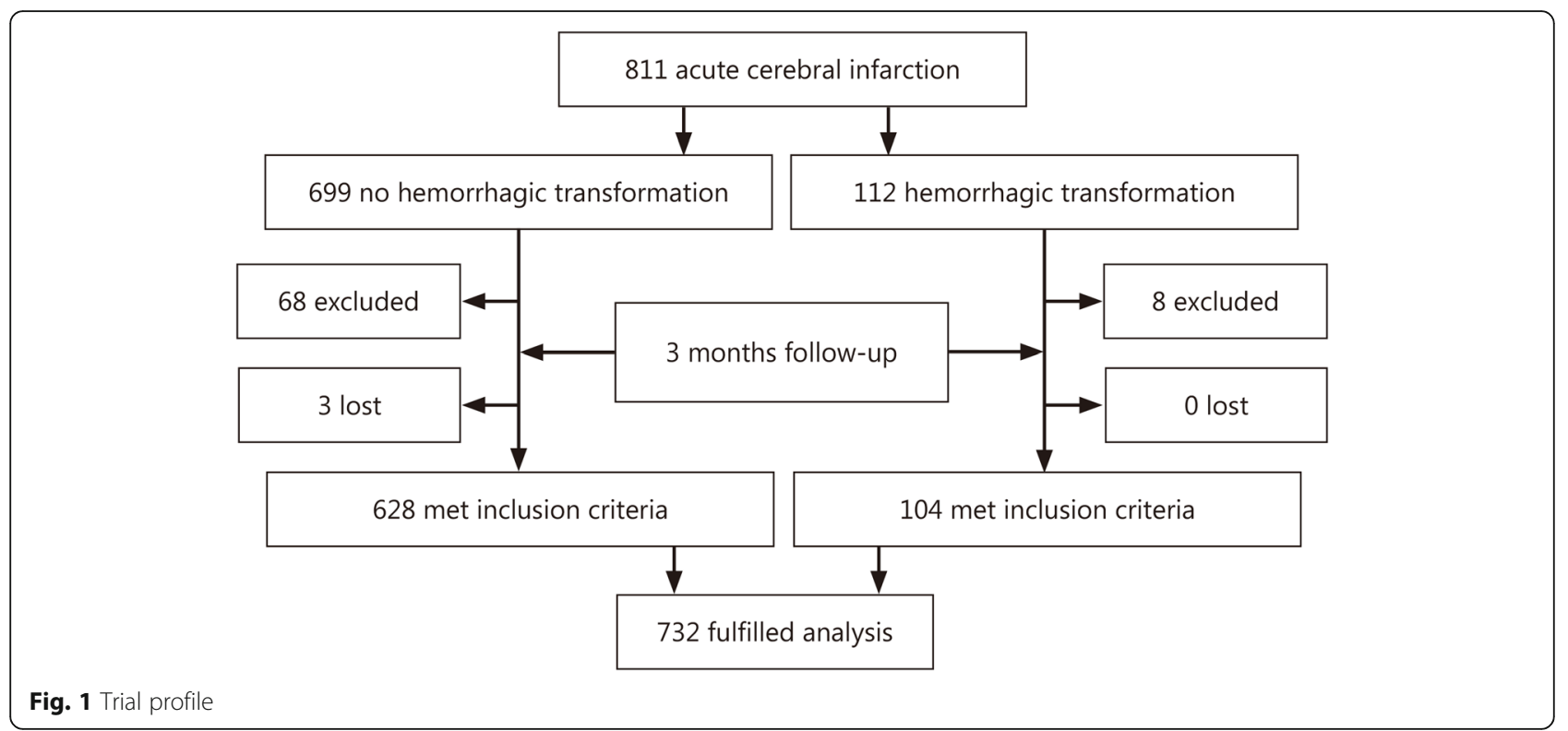

\section{Major treatment}

The percentages of patients treated with rTPA venous thrombolysis, argatroban/low-molecular-weight heparin/ warfarin anticoagulation, double anti-platelet, and SILL between these two groups were not significantly different. The overall venous thrombolysis rate was $12.8 \%$, and the incidences in group A and group B were 12.4\% and $15.4 \%$, respectively (Table 6). The incidence of HT after venous thrombolysis followed by single or double anti-platelet therapy were not significantly different (data not shown).

\section{Analyses of risk factors of HT}

Variable assignment (Table 7) for binary logistic regression. Group was the dependent variable, variable with asterisk $(*)$ was the refrence ctaegory.

The adjusted logistic regression of the maximum likelihood ratio of the risk factors for HT showed that the model had statistical significance $\left(\chi^{2}=143.173, P<0.001\right)$. The Hosmer and Lemeshow goodness-of-fit test had a $P$ value of 0.985 , suggesting that the fit was excellent. The accuracy of prediction was $86.1 \%$. The area under the receiver operating characteristic (ROC) curve was 83.4\% (Fig. 2).

After the confounding factors including age, gender, systolic blood pressure, diabetes, and hypoproteinemia were adjusted, the binary logistic analyses of the risk factors of HT showed that infarct size, cortex involvement and AF were independent risk factors of $\mathrm{HT}$ after ACI (Table 8 ). Because there was a clear collinearity between TC and SILL as well as between TC and history of hyperlipidemia with continuous use of statins, SILL and history of hyperlipidemia were not internalized in the regression. The risk of HT in infarctions with large sizes was 7.158 times higher than that in lacunar infarctions and $34.7 \%$ higher than that in medium-sized infarctions. The risk of HT in infarctions with cortex involvement was 2.083 times higher than that in deep infarctions. The risk of HT in AF patients was 1.989 times higher than that in patients with sinus rhythm. TC was a protective factor of $\mathrm{HT}$; the lower the $\mathrm{TC}$ value was, the higher the risk of HT was. When the normal TC value was reduced by $1 \mathrm{mmol} / \mathrm{L}$, the risk of HT increased by 64.1\%. The influences of rTPA venous thrombolysis and anti-PLT on HT were not statistically significant.

\section{Analyses for outcomes}

(Table 9): the overall mortality was $9.8 \%$, and the mortality in group B was significantly higher than that in group A $(21.2 \%$ vs. $8.0 \%, P<0.001)$. The long-term unfavorable outcome rate was $47.5 \%$, and the unfavorable outcome rate in group $B$ was significantly higher than that in group A (76.0\% vs. $42.8 \%, P<0.001)$.

The adjusted logistic analysis model of the risk factors of a long-term unfavorable outcome was $\chi^{2}=227.147$, $P<0.001$. The goodness-of-fit test of HT showed $P=$ 0.0572 . The prediction accuracy was $72.4 \%$, and the area under the ROC curve was $83.6 \%$ (Fig. 2).

Large infarct size was an independent risk factor for long-term unfavorable outcomes of neurological functions (Table 10). The risk of bad prognosis in large infarct sizes was 12.178 times higher than that in lacunar infarctions. LDL-C was a protective factor. With every $1 \mathrm{mmol} / \mathrm{L}$ of decrease in the normal LDL-C value, the risk of unfavorable outcomes increased by $46.2 \%$. Other confounding factors such as age, gender, AF, systolic blood pressure, diabetes, blood glucose, hypoproteinemia, cortex involvement, TC, 
Table 1 Clinical baseline characteristics

\begin{tabular}{|c|c|c|c|c|}
\hline Characteristics & Total $(n=732)$ & Group A $(n=628)$ & Group B $(n=104)$ & $P$ \\
\hline Age, mean $\pm S D$ & $66.17 \pm 13.19$ & $65.81 \pm 13.14$ & $68.34 \pm 13.34$ & 0.070 \\
\hline$<50$ & $78(10.7)$ & $69(11.0)$ & $9(8.7)$ & 0.697 \\
\hline $50-70$ & $368(50.3)$ & $322(51.3)$ & $46(44.2)$ & 0.561 \\
\hline$>70$ & $286(39.0)$ & $237(37.7)$ & $49(47.1)$ & 0.661 \\
\hline Female/Male $[n(\%)]^{x}$ & 253/479 (34.6/65.4) & $211 / 417(33.6 / 66.4)$ & $42 / 62(40.4 / 59.6)$ & 0.178 \\
\hline HBP, no/yes $[n(\%)]$ & $218 / 514(29.8 / 70.2)$ & $190 / 438(30.3 / 69.7)$ & 28/76 (26.9/73.1) & 0.491 \\
\hline DM, no/yes $[n(\%)]^{H}$ & $487 / 245(66.5 / 33.5)$ & $424 / 204(67.5 / 32.5)$ & $63 / 41(60.6 / 39.4)$ & 0.165 \\
\hline Hlip, no/yes $[n(\%)]^{H}$ & $584 / 148(79.8 / 20.2)$ & 496/132 (79.0/21.0) & $88 / 16(84.6 / 15.4)$ & 0.185 \\
\hline $\mathrm{AF}$, no/yes $[n(\%)]^{H}$ & $604 / 128(82.5 / 17.5)$ & $541 / 87$ (86.1/13.9) & $63 / 41(60.6 / 39.4)$ & $<0.001$ \\
\hline $\mathrm{SBP}(\mathrm{mmHg})$ & $150.35 \pm 23.26$ & $150.54 \pm 23.45$ & $149.20 \pm 22.14$ & 0.586 \\
\hline $\mathrm{DBP}(\mathrm{mmHg})$ & $84.71 \pm 13.49$ & $84.83 \pm 13.73$ & $83.98 \pm 12.00$ & 0.550 \\
\hline NIHSS & $7.05 \pm 6.27$ & $6.50 \pm 6.10$ & $10.39 \pm 6.25$ & $<0.001$ \\
\hline Hypoproteinemia, no/yes $[n(\%)]^{H}$ & 638/94 (87.2/12.8) & $550 / 78(87.6 / 12.4)$ & $88 / 16(84.6 / 15.4)$ & 0.403 \\
\hline Glucose (mmol/L) & $7.33 \pm 3.63$ & $7.19 \pm 3.57$ & $8.16 \pm 3.87$ & 0.011 \\
\hline $\mathrm{TC}(\mathrm{mmol} / \mathrm{L})$ & $4.36 \pm 1.05$ & $4.39 \pm 1.04$ & $4.15 \pm 1.08$ & 0.035 \\
\hline$<3.10$ & $77(10.5)$ & $58(9.3)$ & $19(18.3)$ & 0.857 \\
\hline $3.10-5.70$ & $578(79.0)$ & $507(80.7)$ & $71(68.2)$ & 0.047 \\
\hline$>5.70$ & $77(10.5)$ & $63(10.0)$ & $14(13.5)$ & 0.038 \\
\hline $\mathrm{TG}(\mathrm{mmol} / \mathrm{L})$ & $1.41 \pm 0.87$ & $1.44 \pm 0.90$ & $1.24 \pm 0.70$ & 0.030 \\
\hline$<0.57$ & $47(6.4)$ & $40(6.4)$ & $7(6.7)$ & 0.999 \\
\hline $0.57-1.7$ & $514(70.2)$ & $435(69.2)$ & $79(76.0)$ & 0.044 \\
\hline$>1.7$ & $171(23.4)$ & $153(24.4)$ & $18(17.3)$ & 0.417 \\
\hline $\mathrm{HDL}-\mathrm{C}(\mathrm{mmol} / \mathrm{L})$ & $1.16 \pm 0.33$ & $1.15 \pm 0.33$ & $1.21 \pm 0.32$ & 0.074 \\
\hline$<0.83^{H}$ & $102(13.9)$ & $92(14.6)$ & $10(9.6)$ & 0.192 \\
\hline $0.83-1.96^{H}$ & 614 (83.9) & $523(83.3)$ & $91(87.5)$ & 0.184 \\
\hline$>1.96^{\mathrm{H}}$ & $16(2.2)$ & $13(2.1)$ & $3(2.9)$ & 0.106 \\
\hline LDL-C (mmol/L) & $2.83 \pm 0.94$ & $2.86 \pm 0.92$ & $2.67 \pm 1.01$ & 0.056 \\
\hline$<2.07$ & $152(20.8)$ & $124(19.7)$ & $28(26.9)$ & 0.001 \\
\hline $2.07-3.1$ & $330(45.0)$ & $285(45.4)$ & $45(43.3)$ & 0.443 \\
\hline$>3.1$ & $250(34.2)$ & 219 (34.9) & $31(29.8)$ & 0.761 \\
\hline
\end{tabular}

${ }^{(\mathrm{X})}$. $P$ values were calculated by one-way ANOVA, Pearson's Chi-square test; ${ }^{(H)}$. $P$ values were calculated by the Kruskal-Wallis $H^{(H)}$ test; $H T$ Hemorrhagic transformation postacute cerebral infarction; HBP: History of hypertension; DM: Diabetes mellitus; Hlip Hyperlipidemia; SBP Systolic pressure; DBP Diastolic pressure; NIHSS National Institutes of Health Stroke Scale; TC Total cholesterol; TG Triglyceride; HDL-C High-density lipoprotein cholesterol; LDL-C Low-density lipoprotein cholesterol

TG, anti-PLT and HT had no significant effects on unfavorable outcomes.

\section{Discussion}

The restoration of blood flow perfusion in vessels in ischemic areas after ACI causes different types of secondary HT in different infarct sizes. The CT presentation of $\mathrm{HI}$ is a patchy-shaped, high-density image with a blurred border in infarct sites that can be partially fused. Anatomical results by Teal and Pessin [1] have shown that capillaries or veins in the brains of $\mathrm{HI}$ patients have multifocal blood outflow. It is generally considered that this type of $\mathrm{HI}$ represents erythrocyte exudation through ischemic capillaries rather than vascular rupture. In contrast, $\mathrm{PH}$ is hemorrhage resulting from vascular rupture in ischemic areas caused by reperfusion pressure that can have a space-occupying effect or can cause a leak into the ventricle [5]. The presence of these two types of HT can overlap. Animal studies have shown that the function of the blood-brain barrier is very weak. Cerebral ischemia and reperfusion after ischemia can both cause vascular endothelial injury, resulting in further blood-brain barrier leakage [6].

It has been suggested that $\mathrm{PH}$ is associated with poor prognosis, while $\mathrm{HI}$ is not associated with poor prognosis [7]. Fiorelli et al. [5] found that $\mathrm{HI}$ occurring within 
Table 2 Infarction site $[n(\%)]$

\begin{tabular}{|c|c|c|c|c|c|}
\hline Infarction & & Total & Group A $(n=628)$ & Group B $(n=104)$ & $P$ \\
\hline \multirow[t]{2}{*}{ Site } & ICAs & $526(71.9)$ & $437(69.6)$ & $89(85.6)$ & 0.001 \\
\hline & VBs & $206(28.1)$ & $191(30.4)$ & $15(14.4)$ & \\
\hline \multirow[t]{3}{*}{ Size } & 0 & $468(63.9)$ & $448(71.3)$ & $20(19.2)$ & $<0.001^{a}$ \\
\hline & 1 & $153(20.9)$ & $111(17.7)$ & $42(40.4)$ & $0.074^{b}$ \\
\hline & 2 & $111(15.2)$ & $69(11.0)$ & $42(40.4)$ & $<0.001^{c}$ \\
\hline \multirow[t]{2}{*}{ Cortex } & No & $439(60.0)$ & $418(66.6)$ & $21(20.2)$ & $<0.001$ \\
\hline & Yes & $293(40.0)$ & $210(33.4)$ & $83(79.8)$ & \\
\hline
\end{tabular}

Group A: Acute cerebral infarction without hemorrhagic transformation. Group B: Hemorrhagic transformation postacute cerebral infarction. ICAs: Internal carotid artery system. VBs: Vertebral basilar system. Sites 0,1 , and 2 represent small, medium, and larger areas of infarction, respectively. a. site 0 vs site $1 ;$ b: site 1 vs site 2; c: site 0 vs site 2

$36 \mathrm{~h}$ of ACI did not have an obvious influence on the long-term outcomes, although HI might suggest that at least some ischemic tissues exhibit reperfusion. However, a single-center retrospective study by Krueger et al. [8] on 299 cases of ACI showed that 87 patients had $\mathrm{HT}$; in addition, $\mathrm{PH}$ and $\mathrm{HI}$ were both independently associated with unfavorable neurological outcomes. The multivariate analysis results showed that older age, rTPA venous thrombolysis, and vascular recanalization were associated with HT. High NIHSS scores, high blood glucose, proximal middle cerebral artery occlusion, and a history of hypertension at admission increased the risk of HI, suggesting that HI was not a so-called "benign" imaging sign.

Venous thrombolysis at the acute stage of ACI is an effective method for the restoration of blood reperfusion in ischemic areas; however, it may be concurrent with HT or systemic hemorrhage. A retrospective study by $\mathrm{Xu}$ et al. [9] on 162 patients showed that $12.3 \%$ patients developed HT after rTPA venous thrombolysis. A study by Berger et al. [2] showed that the incidence of HT was $29.5 \%$ in the rTPA venous thrombolysis group and $18.5 \%$ in the placebo group. The difference between these two groups was mainly because the incidence of $\mathrm{PH}$ in the rTPA group was significantly higher than that in the placebo group ( $7.6 \%$ vs. $0.5 \%, P<0.0001)$, while the difference in HI between these two groups was not significant. Chen et al. [10] showed that anti-platelet therapy before

Table 3 AF-associated infarct sizes and HT

\begin{tabular}{|c|c|c|c|c|c|c|c|c|}
\hline \multicolumn{2}{|c|}{ Infarction } & \multicolumn{2}{|l|}{ Total } & \multicolumn{2}{|c|}{ Group A [n (\%)] } & \multicolumn{2}{|c|}{ Group B [n (\%)] } & \multirow[t]{2}{*}{$P$} \\
\hline & & $\mathrm{AF} 0$ & AF 1 & $\mathrm{AF} 0$ & AF 1 & $\mathrm{AF} 0$ & AF 1 & \\
\hline \multirow[t]{3}{*}{ Size } & 0 & $\begin{array}{l}432 \\
(71.6)\end{array}$ & $\begin{array}{l}36 \\
(28.1)\end{array}$ & $\begin{array}{l}415 \\
(76.7)\end{array}$ & $\begin{array}{l}33 \\
(38.0)\end{array}$ & $\begin{array}{l}17 \\
(27.0)\end{array}$ & $\begin{array}{l}3 \\
(7.3)\end{array}$ & 0.193 \\
\hline & 1 & $\begin{array}{l}109 \\
(18.0)\end{array}$ & $\begin{array}{l}44 \\
(33.4)\end{array}$ & $\begin{array}{l}82 \\
(15.2)\end{array}$ & $\begin{array}{l}29 \\
(33.3)\end{array}$ & $\begin{array}{l}27 \\
(42.8)\end{array}$ & $\begin{array}{l}15 \\
(36.6)\end{array}$ & 0.242 \\
\hline & 2 & $\begin{array}{l}63 \\
(10.4)\end{array}$ & $\begin{array}{l}48 \\
(37.5)\end{array}$ & $\begin{array}{l}44 \\
(8.1)\end{array}$ & $\begin{array}{l}25 \\
(28.7)\end{array}$ & $\begin{array}{l}19 \\
(30.2)\end{array}$ & $\begin{array}{l}23 \\
(56.1)\end{array}$ & 0.056 \\
\hline
\end{tabular}

Size: Area of acute cerebral infarction; AF Atrial fibrillation; HT: Hemorrhagic transformation thrombolysis increased the risks of $\mathrm{HT}$ and the 90-d poor prognosis after thrombolysis. A retrospective study by other scholars $[11,12]$ also showed that anti-platelet pretreatment increased the risk of HT after rTPA venous thrombolysis. The 7-d and 14-d NIHSS scores and the 90-d mRS score in the non-anti-platelet group after rTPA venous thrombolysis were all significantly lower than those in the anti-platelet group after thrombolysis. These results suggested that anti-platelet therapy before and after rTPA could both increase the risk of poor prognosis. Our study showed that the rTPA venous thrombolysis rate was $12.8 \%$, with rates in group A and group B of 12.4 and $15.4 \%$, respectively $(P=0.403)$. This result suggests that rTPA venous thrombolysis did not significantly increase the risk of HT.

A prospective study by Paciaroni et al. [13] that included 4 centers and 1125 cases of ACI showed that the incidence of HT was 8.7\%, and the major risk factors of HT included large infarct size, high blood glucose, older age, high NIHSS score at disease onset, and high mean arterial blood pressure. Our study showed that the incidence of HT was $14.2 \%$ (104/732); the onset time was $0-26 \mathrm{~d}$, with a mean onset time of $2 \mathrm{~d}(1,7)$; the percentages of infarctions with large, medium, and small sizes were $15.2,20.9$, and $63.9 \%$, respectively; the corresponding incidence rates of HT were $37.8 \%(42 / 111)$, $27.5 \%(42 / 153)$, and $4.3 \%$ (20/468), respectively; and the incidence of HT in the anterior circulation area (16.9\%) was significantly higher than that in the posterior circulation area (7.3\%). A total of 293 enrolled patients in this

Table 4 HT with cortex involvement in patients with AF-associated cerebral infarction

\begin{tabular}{llllll}
\hline AF & Cortex & Total & Group A[n (\%)] & Group B $[n(\%)]$ & $P$ value \\
\hline 0 & 0 & 397 & $382(96.2)$ & $15(3.8)$ & $<0.001$ \\
& 1 & 207 & $159(76.8)$ & $48(23.2)$ & \\
1 & 0 & 42 & $36(85.7)$ & $6(14.3)$ & 0.003 \\
& 1 & 86 & $51(59.3)$ & $35(40.7)$ & \\
\hline
\end{tabular}

AF Atrial fibrillation. HT Hemorrhagic transformation 
Table 5 NIHSS and HT

\begin{tabular}{llll}
\hline Group & $\mathrm{NIHSS}[n(\%)]$ & & P value \\
\cline { 2 - 3 } & $\leq 4$ & $>4$ & \\
\hline $\mathrm{A}(n=628)$ & $337(53.7)$ & $291(46.3)$ & $<0.001$ \\
A $(n=104)$ & $25(24.0)$ & $79(76.0)$ & \\
\hline
\end{tabular}

NIHSS National Institutes of Health Stroke Scale

study had infarctions with cortex involvement, of which 28.3\% patients had HT. In group B, the percentages of medium-large areas of infarction and infarctions with cortex involvement were 80.8 and $79.8 \%$, respectively, which were both significantly higher than those in group A (28.7 and $33.4 \%$, respectively). The adjusted multivariate regression analysis results showed that the risk of HT with a large infarct size was 7.158 times higher than that with a small infarct size and $34.7 \%$ higher than that with a medium infarct size. The probability of HT in infarctions with cortex involvement was 2.083 times higher than that in deep infarctions. These results indicated that large infarct size and cortex involvement were both important risk factors for HT.

Researches suggested the reasons that large infarctions are prone to HT might be associated with the obvious cerebral edema in infarction lesions and the surroundings that compress peripheral veins to cause blood stagnation and vascular ischemic injury $[14,15]$. During ACI,

Table 6 Major treatment and prognosis for $\mathrm{CH}$ and $\mathrm{CH}$ post $\mathrm{Cl}$ [n (\%)]

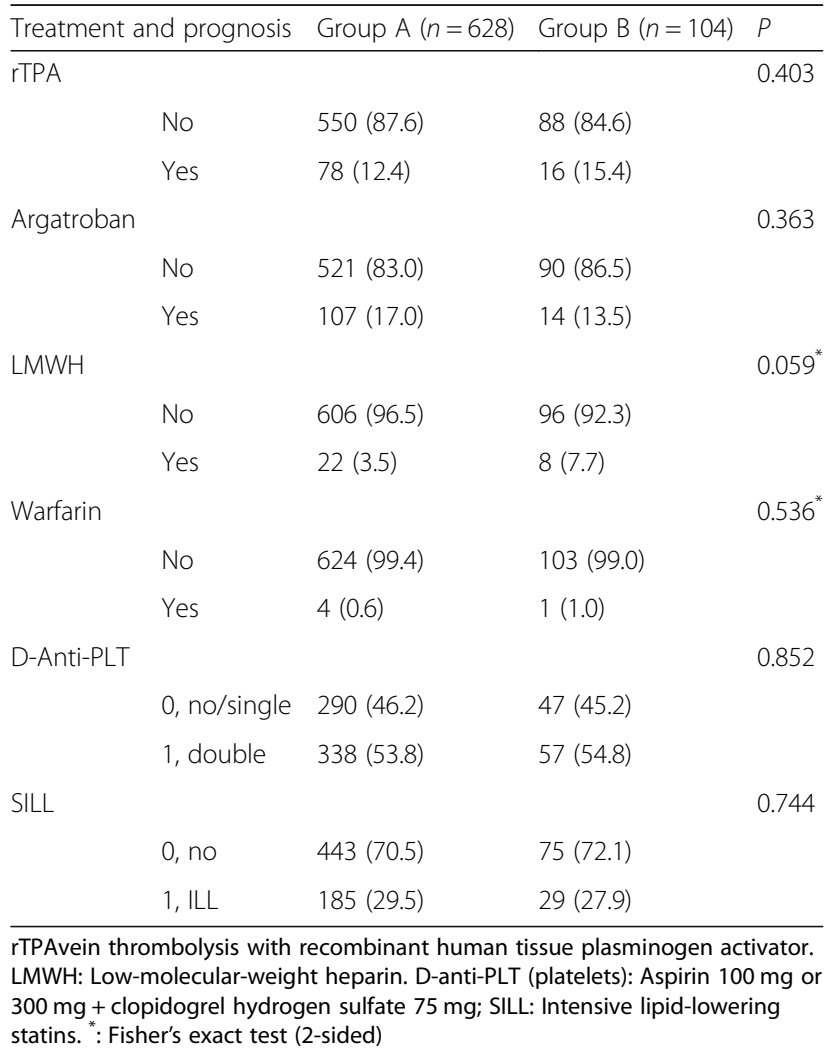

Table 7 Variable assignment

\begin{tabular}{|c|c|c|}
\hline \multicolumn{2}{|l|}{ Variables } & \multirow{2}{*}{$\begin{array}{l}\text { Assignment } \\
0=\mathrm{ACl}, 1^{*}=\mathrm{HT} \text { post } \mathrm{ACl}\end{array}$} \\
\hline Group & $\begin{array}{l}\text { Group (dependent } \\
\text { variable) }\end{array}$ & \\
\hline NIHSS & $\begin{array}{l}\text { National Institutes } \\
\text { of Health Stroke Scale }\end{array}$ & $0^{*} \leq 4,1>4$ \\
\hline Sex & Gender & $0=$ female, $1^{*}=$ male \\
\hline Age (year) & Age & $0^{*}<50,1=50$ to $70,2>70$ \\
\hline SBP & Systolic blood pressure & continuous variable \\
\hline DM & Diabetes mellitus history & $0=$ no, $1^{*}=$ yes \\
\hline Hypopro & hypoproteinemia & $0=$ no, $1^{*}=$ yes \\
\hline AF & Atrial fibrillation history & $0=$ no, $1^{*}=$ yes \\
\hline $\mathrm{TC}(\mathrm{mmol} / \mathrm{L})$ & Total cholesterol & $0^{*}=$ low, $1=$ normal $; 2=$ high \\
\hline TG $(\mathrm{mmol} / \mathrm{L})$ & Triglyceride & $0^{*}=$ low, $1=$ normal $; 2=$ high \\
\hline LDL-C (mmol/L) & $\begin{array}{l}\text { Low-density lipoprotein } \\
\text { cholesterol }\end{array}$ & $0^{*}=$ low, $1=$ normal $; 2=$ high \\
\hline $\mathrm{HDL}-\mathrm{C}(\mathrm{mmol} / \mathrm{L})$ & $\begin{array}{l}\text { High-density lipoprotein } \\
\text { cholesterol }\end{array}$ & $\begin{array}{l}0^{*}<0.86,1=0.8-1.96 \\
2>1396\end{array}$ \\
\hline Site & Infarction site & $\begin{array}{l}0=\text { Anterior, } 1^{*}=\text { posterior } \\
\text { circulation }\end{array}$ \\
\hline Size & Infarct size & $0^{*}=$ small, $1=$ middle, $2=$ large \\
\hline Cortex & Cortex involvement & $0^{*}=$ no, $1=$ yes \\
\hline anti-PLT & Anti-platelet & $\begin{array}{l}0=\mathrm{no}^{*}, 1=\text { aspirin } 100 \mathrm{mg} \text { or } \\
\text { clopidogrel } 75 \mathrm{mg} ; 2=\text { aspirin } \\
100 \mathrm{mg}+\text { clopidogrel } 75 \mathrm{mg} \\
3=\text { aspirin } 300 \mathrm{mg} \text { or } \\
\text { clopidogrel } 300 \mathrm{mg}\end{array}$ \\
\hline
\end{tabular}

*. Reference category. ACl (acute cerebral infarction). HT (hemorrhagic transformation post ACl). NIHSS (National Institutes of Health Stroke Scale) 
Table 8 Adjusted logistic analysis for risk factors of HT

\begin{tabular}{|c|c|c|c|c|c|c|}
\hline \multirow[t]{2}{*}{ Item } & \multirow[t]{2}{*}{ B } & \multirow[t]{2}{*}{$d f$} & \multirow[t]{2}{*}{ Adjusted $O R$} & \multicolumn{2}{|l|}{$95 \% \mathrm{Cl}$} & \multirow[t]{2}{*}{$P$ value } \\
\hline & & & & Lower & Upper & \\
\hline Age & & 2 & & & & 0.727 \\
\hline Age (1) & -0.209 & 1 & 0.811 & 0.341 & 1.930 & 0.636 \\
\hline Age (2) & -0.006 & 1 & 0.994 & 0.403 & 2.451 & 0.989 \\
\hline Sex & 0.144 & 1 & 1.155 & 0.674 & 1.978 & 0.600 \\
\hline DM & 0.476 & 1 & 1.609 & 0.959 & 2.699 & 0.072 \\
\hline $\mathrm{AF}$ & 0.687 & 1 & 1.989 & 1.089 & 3.632 & 0.025 \\
\hline SBP & 0.000 & 1 & 1.000 & 0.989 & 1.012 & 0.937 \\
\hline Hypoproteinemia & -0.201 & 1 & 0.818 & 0.408 & 1.641 & 0.572 \\
\hline Site & -0.563 & 1 & 0.569 & 0.297 & 1.093 & 0.090 \\
\hline Size & & 2 & & & & 0.000 \\
\hline Size(1) & 1.670 & 1 & 5.310 & 2.581 & 10.925 & 0.000 \\
\hline Size(2) & 1.968 & 1 & 7.158 & 3.020 & 16.962 & 0.000 \\
\hline Cortex & 0.734 & 1 & 2.083 & 1.024 & 4.236 & 0.043 \\
\hline TC & & 2 & & & & 0.020 \\
\hline $\mathrm{TC}(1)$ & -1.025 & 1 & 0.359 & 0.136 & 0.944 & 0.038 \\
\hline TC (2) & -0.191 & 1 & 0.826 & 0.223 & 3.057 & 0.775 \\
\hline TG & & 2 & & & & 0.874 \\
\hline TG (1) & 0.264 & 1 & 1.303 & 0.474 & 3.582 & 0.609 \\
\hline TG (2) & 0.284 & 1 & 1.328 & 0.408 & 4.329 & 0.638 \\
\hline $\mathrm{HDL}$ & & 2 & & & & 0.393 \\
\hline $\mathrm{HDL}(1)$ & 0.421 & 1 & 1.524 & 0.680 & 3.418 & 0.306 \\
\hline HDL (2) & -0.291 & 1 & 0.747 & 0.140 & 3.993 & 0.733 \\
\hline LDL & & 2 & & & & 0.368 \\
\hline LDL (1) & 0.313 & 1 & 1.367 & 0.608 & 3.075 & 0.449 \\
\hline LDL (2) & -0.131 & 1 & 0.877 & 0.349 & 2.207 & 0.781 \\
\hline Anti-PLT & & 3 & & & & 0.058 \\
\hline Anti-PLT (1) & -0.327 & 1 & 0.721 & 0.340 & 1.528 & 0.393 \\
\hline Anti-PLT (2) & 0.558 & 1 & 1.747 & 0.959 & 3.182 & 0.068 \\
\hline Anti-PLT (3) & 0.609 & 1 & 1.838 & 0.748 & 4.520 & 0.185 \\
\hline rTPA & -0.022 & 1 & 0.978 & 0.492 & 1.945 & 0.950 \\
\hline Constant & -3.074 & 1 & 0.046 & & & 0.012 \\
\hline
\end{tabular}

METHOD $=$ ENTER, Entry $=0.05$, Removal $=0.10$; Age. $0<50,1=50$ to 70 , $2>70$ years; Sex. $0=\mathrm{F}, 1=\mathrm{M}$; $D M$ Diabetes mellitus, $A F$ Atrial fibrillation, SBP Systolic blood pressure; Site. $0=$ Anterior, $1=$ posterior circulation; Size. $0=$ small, $1=$ middle, $2=$ large of infarction; Cortex. $0=$ untouched, $1=$ involved; TC. Total cholesterol, $0=$ lower than normal, $1=$ normal, $2=$ higher than normal; AntiPLT. Platelets, $0=$ no, $1=$ aspirin $100 \mathrm{mg}$ or clopidogrel $75 \mathrm{mg}, 2=$ aspirin $100 \mathrm{mg}+$ clopidogrel $75 \mathrm{mg}, 3$ = aspirin $300 \mathrm{mg}$ or clopidogrel $300 \mathrm{mg}$; TG Triglyceride, HDL High density lipoprotein cholesterol, LDL Low density lipoprotein cholesterol, rTPA Recombinant human tissue plasminogen activator

ischemia and hypoxia in the infarct site cause degeneration of the vascular intima and increase the vascular permeability. When edema subsides, and the collateral circulation is established, vascular reperfusion occurs at the infarct sites to cause HT under the impact of blood [1]. Abundant collateral circulation is present in the
Table 9 Prognosis of the two groups[n (\%)]

\begin{tabular}{lllll}
\hline \multicolumn{2}{ll}{ Prognosis } & Group A $(n=628)$ & Group B $(n=104)$ & $P$ \\
\hline Died & & & & $<0.001$ \\
& No & $578(92.0)$ & $82(78.8)$ & \\
& Yes & $50(8.0)$ & $22(21.2)$ & $<0.001$ \\
mRS & & & & \\
& 0-2 (good) & $359(57.2)$ & $25(24.0)$ & \\
& $>2$ (bad) & $269(42.8)$ & $79(76.0)$ & \\
\hline
\end{tabular}

mRS Modified Rankin scale

cerebral cortex; therefore, reperfusion injuries in infarctions with cortex involvement are more severe. Furthermore, animal experiment by $\mathrm{Hu}$ et al. [16] found that blood-brain barrier destruction and cerebrovascular self-regulation repair functional impairments caused by ischemia-induced abnormal cellular energy metabolism further induce HT during reperfusion or establishment of the collateral circulation. Infarction in cortical white matter involves the peripheral blood supply areas of the artery; therefore, collateral circulation is usually not sufficient, and lacunar infarction is mainly present. Thus, the incidence of HI is lower; even though HI is present, it mainly includes focal hemorrhage.

The incidence of AF in this study was $17.5 \%$; the incidence in group $B$ was significantly higher than that in group A $(39.4 \%$ vs. $13.9 \%, P<0.001)$. The percentages of large, medium, and small sizes of ACI in AF patients were $37.5 \%, 34.4 \%$, and $28.1 \%$, respectively, and the corresponding incidence rates of HT were $56.1 \%, 36.6 \%$, and $7.3 \%$, respectively, which were not significantly different from those in patients with sinus rhythm. However, the incidence of $\mathrm{HT}$ in patients with AF-associated ACI with cortex involvement was significantly higher than that in patients without cortex involvement $(40.7 \%$ vs. $14.3 \%, P=0.003)$. The incidence of HT in patients with sinus rhythm-associated ACI with cortex involvement $(23.2 \%)$ was also significantly higher than that in patients without cortex involvement (3.8\%). The adjusted multivariate analysis results showed that AF was another important risk factor for HT.

High blood glucose is also closely associated with HI. High blood glucose causes mitochondrial dysfunction, increases the activity of matrix metalloproteinases, and promotes cell apoptosis to cause HT [17]. Studies by Okamura et al. [18] in a middle cerebral artery ischemia reperfusion model in high blood glucose rats have suggested that high blood glucose promotes enlargement of the infarction lesions, aggravates cerebral edema, and increases the risk of HT through aggravation of energy metabolism disorders. Control of blood glucose by sulfonylureas could significantly reduce HT [19]. The univariate analysis results in this study showed that the percentages of patients with a history of diabetes mellitus 
Table 10 Adjusted logistic analysis of the risk factors of long-term unfavorable outcomes

\begin{tabular}{|c|c|c|c|c|c|c|}
\hline \multirow[t]{2}{*}{ Item } & \multirow[t]{2}{*}{ B } & \multirow[t]{2}{*}{$d f$} & \multirow[t]{2}{*}{ Adjusted $O R$} & \multicolumn{2}{|l|}{$95 \% \mathrm{Cl}$} & \multirow[t]{2}{*}{$P$ value } \\
\hline & & & & Lower & Upper & \\
\hline Age & & 2 & & & & 0.118 \\
\hline Age (1) & 0.176 & 1 & 1.192 & 0.660 & 2.154 & 0.561 \\
\hline Age (2) & 0.524 & 1 & 1.689 & 0.906 & 3.149 & 0.099 \\
\hline Sex & -0.064 & 1 & 0.938 & 0.638 & 1.377 & 0.743 \\
\hline DM & -0.284 & 1 & 0.753 & 0.515 & 1.101 & 0.143 \\
\hline AF & 0.228 & 1 & 1.256 & 0.732 & 2.153 & 0.408 \\
\hline SBP & 0.002 & 1 & 1.002 & 0.994 & 1.009 & 0.648 \\
\hline Hypoproteinemia & 0.126 & 1 & 1.135 & 0.654 & 1.970 & 0.653 \\
\hline Site & -0.030 & 1 & 0.970 & 0.660 & 1.426 & 0.877 \\
\hline Size & & 2 & & & & 0.000 \\
\hline Size(1) & 1.278 & 1 & 3.588 & 2.176 & 5.915 & 0.000 \\
\hline Size(2) & 2.500 & 1 & 12.178 & 5.390 & 27.516 & 0.000 \\
\hline Cortex & 0.313 & 1 & 1.367 & 0.862 & 2.169 & 0.184 \\
\hline TC & & 2 & & & & 0.062 \\
\hline $\mathrm{TC}(1)$ & 0.701 & 1 & 2.016 & 0.940 & 4.325 & 0.072 \\
\hline $\mathrm{TC}(2)$ & 1.204 & 1 & 3.335 & 1.227 & 9.065 & 0.018 \\
\hline TG & & 2 & & & & 0.032 \\
\hline TG (1) & -0.392 & 1 & 0.676 & 0.321 & 1.423 & 0.302 \\
\hline TG (2) & -0.929 & 1 & 0.395 & 0.170 & 0.919 & 0.031 \\
\hline $\mathrm{HDL}$ & & 2 & & & & 0.985 \\
\hline $\mathrm{HDL}(1)$ & 0.047 & 1 & 1.048 & 0.606 & 1.813 & 0.867 \\
\hline HDL (2) & 0.012 & 1 & 1.012 & 0.250 & 4.098 & 0.986 \\
\hline LDL & & 2 & & & & 0.030 \\
\hline LDL (1) & -0.620 & 1 & 0.538 & 0.300 & 0.964 & 0.037 \\
\hline LDL (2) & -0.151 & 1 & 0.860 & 0.456 & 1.620 & 0.640 \\
\hline Anti-PLT & & 3 & & & & 0.110 \\
\hline Anti-PLT (1) & 0.068 & 1 & 1.071 & 0.638 & 1.797 & 0.796 \\
\hline Anti-PLT (2) & -0.355 & 1 & 0.701 & 0.452 & 1.088 & 0.113 \\
\hline Anti-PLT (3) & -0.554 & 1 & 0.575 & 0.296 & 1.116 & 0.102 \\
\hline Group & 0.528 & 1 & 1.696 & 0.942 & 3.054 & 0.078 \\
\hline Constant & -1.538 & 1 & 0.215 & & & 0.084 \\
\hline
\end{tabular}

METHOD $=$ ENTER, Entry $=0.05$, Removal $=0.10$; Age. $0<50,1=50$ to 70, $2>$ 70 years; Sex. $0=F, 1=M$; Site $0=$ anterior, $1=$ posterior circulation; Size. $0=$ small, $1=$ middle, 2 = large of infarction; Cortex. $0=$ untouched, $1=$ involved; TC. Total cholesterol, $0=$ lower than normal, $1=$ normal, $2=$ higher than normal; Anti-PLT. Platelets $0=$ no, $1=$ aspirin $100 \mathrm{mg}$ or clopidogrel $75 \mathrm{mg}, 2=$ aspirin $100 \mathrm{mg}$ + clopidogrel $75 \mathrm{mg}, 3=$ aspirin $300 \mathrm{mg}$ or clopidogrel $300 \mathrm{mg}$; Group. $0=A$, no hemorrhagic transformation; $1=B$, hemorrhagic transformation; DM. Diabetes; AF. Atrial fibrillation; SBP. Systolic blood pressure; Hypopro. Hhypoproteinemia; TG. Triglyceride; HDL. High density lipoprotein cholesterol; LDL. Low density lipoprotein cholesterol

were not significantly different between these two groups; however, blood glucose at admission was significantly higher in group B than in group A. The adjusted multivariate analysis results showed that blood glucose at admission did not enter the HT risk factor model $(O R=$ $1.609,95 \%$ CI $0.959-2.699$ ) or the long-term poor prognosis model $(O R=0.753,95 \%$ CI $0.515-1.101)$. This result suggests that neither the risk of HT nor bad outcomes after ACI was obviously associated with hyperglycemia.

With the extensive application of lipid-lowering statin drugs, the associations between blood lipids and stroke subtypes has received more attention. The study of Zhang et al. [20] showed that when the TC level increased by $1 \mathrm{mmol} / \mathrm{L}$, the corresponding risk of ischemic stroke increased $25 \%$. One meta-analysis by Zhang et al. [21] of 26 randomized trials about intensive lowering LDL-C including 10 participants showed that with every $1 \mathrm{mmol} / \mathrm{L}$ reduction in LDL-C, the annual incidences of heart attack, revascularization, and ischemic stroke were reduced by over a fifth.

Another meta-analysis of 31 randomized controlled trials on statin therapy and the risk of intracerebral hemorrhage by McKinney and Kostis [22] showed that there were no significant differences in the incidence of ICH between the group of 91,588 subjects using statins and the group of 91,215 control subjects $(O R=1.08$; $95 \%$ CI $0.88-1.32 ; P=0.47$ ). This suggests that the ICH risk was not related to the degree of LDL-C reduction. Moreover, they found that the incidence of total stroke $(O R=0.84 ; 95 \%$ CI $0.78-0.91 ; P<0.0001)$ and all-cause mortality $(O R=0.92 ; 95 \%$ CI $0.87-0.96 ; P=0.0007)$ were significantly reduced in the statin therapy group. A prospective study by Rocco et al. [23] showed that blood lipid levels and prior statin use were not associated with HT, functional outcomes, or mortality at 3 months. However, Amarenco et al. showed [24] that ACI or transient cerebral ischemic attack treated with atorvastatin $(80 \mathrm{mg} / \mathrm{d})$ intensive lipid-lowering therapy increased the risk of HT 66\% over that of the control group. The meta-analysis of Wang et al. [25] that included 23 studies showed that TC and cerebral hemorrhage showed a negative level-effect association (with every $1 \mathrm{mmol} / \mathrm{L}$ increase in TC, $O R=0.85$, 95\% CI 0.80-0.91). Sturgeon et al. [26] also showed that LDL-C negatively correlated with cerebral hemorrhage. Yang et al. [27] studied 348 cases of ACI and showed that the levels of TG, HDL-C, and LDL-C in 10.1\% (35 cases) of patients who developed HT were all lower than those in the control group. A retrospective analysis by D'Amelio et al. [28] on 240 patients with anterior circulation ACI showed that the risk of developing HT in the low TC group $(1.95 \pm 0.54 \mathrm{mmol} / \mathrm{L})$ was 2.8 times higher than that in the high TC group $(2.25 \pm 0.48 \mathrm{mmol} / \mathrm{L})$ and that the risk of developing HT in the low LDL-C group (2.66 \pm $0.88 \mathrm{mmol} / \mathrm{L}$ ) was 5.0 times higher than that in the high LDL-C group ( $3.13 \pm 0.97 \mathrm{mmol} / \mathrm{L})$. Olsen et al. [29] found that higher TC levels have been associated with better short-term outcomes after ACI, independently of subtype, vascular territory, age, and glycemia. 
The univariate analysis results in this study showed that the TC and TG levels in group B were significantly lower than those in group $\mathrm{A}$ and that the percentage of low LDL-C cases in group B was significantly higher than that in group A $(26.9 \%$ vs. $19.7 \%, P=0.001)$. The adjusted multivariate analysis results showed that TC was a protective factor for HT; with every $1 \mathrm{mmol} / \mathrm{L} \mathrm{re}$ duction in normal TC levels, the risk of HT increased $64.1 \%$. This suggests that low TC levels were associated with HT. Additionally, the adjusted analysis on the risk factors of poor prognosis showed that the risk of poor prognosis was increased in patients with lower levels of LDL-C; for every $1 \mathrm{mmol} / \mathrm{L}$ reduction in normal LDL-C levels, the risk of an unfavorable outcome increased $46.2 \%$.

The exact pathogenesis of lipid-related HT after ACI is not yet established. Adequate lipid levels affect the vasculature and are essential for maintaining normal membrane fluidity and the integrity of vessels and their resistance to rupture [30, 31]. Extensively low lipid levels will destroy the integrity of the small vessels and result in blood extravasation over the unstable integrity of endothelial cells in the small cerebral vessels [32]. Bang et al. [33] found low LDL-C levels were independently associated with blood brain barrier permeability derangements, which increased the propensity for hemorrhagic transformation after ACI. An early study by Konishi et al. [34] showed that lower TC resulted in a weakened endothelium that more readily led to arterial fragility, hemorrhage, or slower repair after small hemorrhages. However, this study was based on an association between TC in the range of 3.10$4.65 \mathrm{mmol} / \mathrm{L}$, compared to $>4.65 \mathrm{mmol} / \mathrm{L}$ in this study, and on different stroke subtypes, irrespective of cofactors such as age and hypertension.

Our data analysis shows the time of HT onset was mainly within $2 \mathrm{~d}(1,7)$. An explanation for the relationship between $2 \mathrm{~d}$ of SILL and the occurrence of HT might be that the dose of statins required by Chinese patients to achieve the same LDL-C target was low, and the Chinese were less tolerant to the same dose of statins $[35,36]$. However, all of our patients had history of hyperlipidemia and continuous use of statins prior to the current ACI, and a caveat of our research is that we did not repeatedly test blood lipids, such as at 2 or $7 \mathrm{~d}$ after SILL, to compare the changes of all patients' lipid levels.

A study by D'Amelio et al. [37] showed that the longterm mortality after 3 months of ACI was $12.1 \%$ (28/232). The multivariate analysis results showed that large infarct size $(H R=2.7,95 \%$ CI 1.2-6.0 $)$ and HT $(H R=2.3,95 \% \mathrm{CI}$ 1.0-5.4) were independent risk factors of long-term poor prognosis. The adjusted multivariate logistic analysis on long-term poor prognosis of ACI showed that large infarct size was an independent risk factor of long-term unfavorable outcomes in neurologic function. The risk of unfavorable outcome in patients with large infarct size was 12.178 times higher than that in patients with small infarct sizes.

\section{Conclusion}

In summary, HT generally occurs $2 \mathrm{~d}(1,7)$ after ACI. Large infarct size, cortex involvement, AF and lower TC are independent risk factors of HT. With every $1 \mathrm{mmol} / \mathrm{L}$ reduction in normal TC levels, the risk of HT increases by $64.1 \%$. The risk factors of poor prognosis include large infarct size and lower LDL-C. With every $1 \mathrm{mmol} / \mathrm{L}$ reduction in the normal LDL-C level, the risk of unfavorable outcomes increases by $46.2 \%$. Therefore, for ACI patients with AF, large areas of cortex involvement, or low levels of TC, the risk of HT should be vigilantly monitored. Major therapies with intravenous rTPA, intensive lipid-lowering statins and anti-platelets were not significantly related to $\mathrm{HT}$ or long-term poor prognosis after ACI.

The limitations of this study are that it is not a prospective case-control study and that the major observation indicator, blood lipids, was measured from fasting blood collected in the morning after admission. In addition, most patients did not receive blood lipid follow-up at the acute stage; therefore, group statistics could not be performed on repeatedly measured blood lipid levels after drug administration.

\section{Abbreviations \\ ACl: Acute cerebral infarction; AF: Atrial fibrillation; CT: Computed tomography; HI: Hemorrhagic infarction; HT: Hemorrhagic transformation; LDL-C: Low-density lipoprotein cholesterol; MRI: Magnetic resonance imaging; mRS: Modified Rankin Scale; NIHSS: National Institute of Health Stroke Scale; PH: Parenchymal hematoma; rTPA: Recombinant tissue-type plasminogen activator; SILL: Intensive lipid-lowering statins \\ Funding \\ None. \\ Availability of data and materials \\ Raw data was not approved for release by the PLA Army General Hospital. Please contact the author for data requests.}

\section{Authors' contributions}

$G L$ collected data, assisted with the study design and wrote and edited the manuscript. ZXX, HX W, NL, WW, HYH, and WWZ collected data and assisted with the study design. GQW conceived the study and edited the manuscript. All authors read and approved the final manuscript.

Ethics approval and consent to participate

This study was approved by the Medical Ethics Committee of the PLA Army General Hospital.

Consent for publication

Not applicable.

\section{Competing interests}

The authors declare that they have no competing interests.

\section{Author details}

'Department of General Surgery, 309 Hospital of Chinese People's Liberation Army, Beijing 100091, China. ²Department of Neurology, Chinese PLA Army General Hospital, Beijing 100700, China. 
Received: 15 March 2018 Accepted: 2 January 2019

Published online: 22 January 2019

\section{References}

1. Teal PA, Pessin MS. Hemorrhagic transformation. The spectrum of ischemiarelated brain hemorrhage. Neurosurg Clin N Am. 1992;3(3):601-10.

2. Berger C, Fiorelli $M$, Steiner $T$, Schäbitz WR, Bozzao L, Bluhmki E, et al. Hemorrhagic transformation of ischemic brain tissue asymptomatic or symptomatic. Stroke. 2001;32(6):1330-5.

3. Jauch EC, Saver JL, Adams HP Jr, Bruno A, Connors JJ, Demaerschalk BM, et al. Guidelines for the early management of patients with acute ischemic stroke: a guideline for healthcare professionals from the American Heart Association/American Stroke Association. Stroke. 2013;44(3):870-947.

4. Adams HP Jr, Bendixen BH, Kappelle LJ, Biller J, Love BB, Gordon DL, et al. Classification of subtype of acute ischemic stroke. Definitions for use in a multicenter clinical trial. TOAST. Trial of org 10172 in acute stroke treatment. Stroke. 1993;24(1):35-41.

5. Fiorelli M, Bastianello S, von Kummer R, del Zoppo GJ, Larrue V, Lesaffre E, et al. Hemorrhagic transformation within 36 hours of a cerebral infarct: relationships with early clinical deterioration and 3-month outcome in the European cooperative acute stroke study I (ECASS I) cohort. Stroke. 1999;30(11):2280-4

6. Krueger M, Bechmann I, Immig K, Reichenbach A, Härtig W, Michalski D. Blood-brain barrier breakdown involves four distinct stages of vascular damage in various models of experimental focal cerebral ischemia. J Cereb Blood Flow Metab. 2015;35(2):292-303.

7. Ho BL, Chen CF, Lin RT, Liu CK, Chao AC. Clinical implication of hemorrhagic transformation in ischemic stroke patients treated with recombinant tissue plasminogen activator. Neurol Sci. 2016;37(11):1799-805.

8. Kaesmacher J, Kaesmacher M, Maegerlein C, Zimmer C, Gersing AS, Wunderlich S, et al. Hemorrhagic transformations after thrombectomy: risk factors and clinical relevance. Cerebrovasc Dis. 2017;43(5-6):294-304.

9. Xu X, Li C, Wan T, Gu X, Zhu W, Hao J, et al. Risk factors for hemorrhagic transformation after intravenous thrombolysis in acute cerebral infarction: a retrospective single-center study. World Neurosurg. 2017;101:155-60.

10. Chen S, Lu X, Zhang W, Han Z, Yang W, Huang X, et al. Does prior antiplatelet treatment increase the risk of hemorrhagic transformation and unfavorable outcome on day 90 after intravenous thrombolysis in acute ischemic stroke patients? J Stroke Cerebrovasc Dis. 2016;25(6):1366-70.

11. Dorado L, Millán M, de la Ossa NP, Guerrero C, Gomis M, López-Cancio E, et al. Influence of antiplatelet pre-treatment on the risk of intracranial haemorrhage in acute ischaemic stroke after intravenous thrombolysis. Eur J Neurol. 2010;17(2):301-6.

12. Lu J, Li YH, Li YD, Li MH, Zhao JG, Chen SW. The clinical value of antiplatelet therapy for patients with hemorrhage after thrombolysis based on susceptibility-weighted imaging: a prospective pilot study. Eur J Radiol. 2012;81(12):4094-8.

13. Paciaroni M, Agnelli G, Corea F, Ageno W, Alberti A, Lanari A, et al. Early hemorrhagic transformation of brain infarction: rate, predictive factors, and influence on clinical outcome: results of a prospective multicenter study. Stroke. 2008;39(8):2249-56.

14. Krueger M, Härtig W, Frydrychowicz C, Mueller WC, Reichenbach A, Bechmann I, et al. Stroke-induced blood-brain barrier breakdown along the vascular tree-no preferential affection of arteries in different animal models and in humans. J Cereb Blood Flow Metab. 2017;37(7):2539-54.

15. Khatri R, McKinney AM, Swenson B, Janardhan V. Blood-brain barrier reperfusion injury, and hemorrhagic transformation in acute ischemic stroke. Neurology. 2012;79(13 Suppl 1):S52-7.

16. Hu Q, Manaenko A, Bian H, Guo Z, Huang JL, Guo ZN, et al. Hyperbaric oxygen reduces infarction volume and hemorrhagic transformation through ATP/NAD ${ }^{+}$/Sirt1 pathway in hyperglycemic middle cerebral artery occlusion rats. Stroke. 2017;48(6):1655-64.

17. Mishiro K, Imai T, Sugitani S, Kitashoji A, Suzuki Y, Takagi T, et al. Diabetes mellitus aggravates hemorrhagic transformation after ischemic stroke via mitochondrial defects leading to endothelial apoptosis. PLoS One. 2014;9(8):e103818.

18. Okamura K, Tsubokawa T, Johshita H, Miyazaki H, Shiokawa $Y$. Edaravone, a free radical scavenger, attenuates cerebral infarction and hemorrhagic infarction in rats with hyperglycemia. Neurol Res. 2014; 36(1):65-9.
19. Kunte H, Busch MA, Trostdorf K, Vollnberg B, Harms L, Mehta Rl, et al. Hemorrhagic transformation of ischemic stroke in diabetics on sulfonylureas. Ann Neurol. 2012;72(5):799-806.

20. Zhang X, Patel A, Horibe H, Wu Z, Barzi F, Rodgers A, et al. Cholesterol, coronary heart disease, and stroke in the Asia Pacific region. Int J Epidemiol. 2003;32(4):563-72.

21. Cholesterol Treatment Trialists' (CTT) Collaboration, Baigent C, Blackwell L, Emberson J, Holland LE, Reith C, et al. Efficacy and safety of more intensive lowering of LDL-C cholesterol: a meta-analysis of data from 170,000 participants in 26 randomised trials. Lancet. 2010;376(9753):1670-81.

22. McKinney JS, Kostis WJ. Statin therapy and the risk of intracerebral hemorrhage: a meta-analysis of 31 randomized controlled trials. Stroke. 2012;43(8):2149-56.

23. Rocco A, Sykora M, Ringleb P, Diedler J. Impact of statin use and lipid profile on symptomatic intracerebral haemorrhage, outcome and mortality after intravenous thrombolysis in acute stroke. Cerebrovasc Dis. 2012;33(4): 362-8.

24. Amarenco P, Bogousslavsky J, Callahan A 3rd, Goldstein LB, Hennerici M, Rudolph $A E$, et al. High-dose atorvastatin after stroke or transient ischemic attack. N Engl J Med. 2006;355(6):549-59.

25. Wang X, Dong Y, Qi X, Huang C, Hou L. Cholesterol levels and risk of hemorrhagic stroke: a systematic review and meta-analysis. Stroke. 2013; 44(7):1833-9.

26. Sturgeon JD, Folsom AR, Longstreth WT Jr, Shahar E, Rosamond WD, Cushman M. Risk factors for intracerebral hemorrhage in a pooled prospective study. Stroke. 2007;38(10):2718-25.

27. Yang $N$, Lin M, Wang BG, Zeng WY, He YF, Peng HY. Low level of lowdensity lipoprotein cholesterol is related with increased hemorrhagic transformation after acute ischemic cerebral infarction. Eur Rev Med Pharmacol Sci. 2016;20(4):673-8.

28. D'Amelio M, Terruso V, Famoso G, Ragonese P, Aridon P, Savettieri G. Cholesterol levels and risk of hemorrhagic transformation after acute ischemic stroke. Cerebrovasc Dis. 2011;32(3):234-8.

29. Olsen TS, Christensen RH, Kammersgaard LP, Andersen KK. Higher total serum cholesterol levels are associated with less severe strokes and lower all-cause mortality: ten-year follow-up of ischemic strokes in the Copenhagen stroke study. Stroke. 2007;38(10):2646-51.

30. Yaghi S, Elkind MS. Lipids and cerebrovascular disease: research and practice. Stroke. 2015;46(11):3322-8.

31. Bang OY, Saver JL, Liebeskind DS, Starkman S, Villablanca P, Salamon N, et al. Cholesterol level and symptomatic hemorrhagic transformation after ischemic stroke thrombolysis. Neurology. 2007;68(10):737-42.

32. Reed DM. The paradox of high risk of stroke in population with low risk of coronary heart disease. Am J Epidemiol. 1990;131(4):579-88.

33. Bang OY, Saver JL, Alger JR, Shah SH, Buck BH, Starkman S, et al. Patterns and predictors of blood-brain barrier permeability derangements in acute ischemic stroke. Stroke. 2009;40(2):454-61.

34. Konishi M, Iso H, Komachi Y, lida M, Shimamoto T, Jacobs DR Jr, et al. Associations of serum total cholesterol, different types of stroke, and stenosis distribution of cerebral arteries. The Akita Pathology Study Stroke. 1993;24(7):954-64

35. Zhao SP, Yu BL, Peng DQ, Huo Y. The effect of moderate-dose versus double-dose statins on patients with acute coronary syndrome in China: results of the CHILLAS trial. Atherosclerosis. 2014;233(2):707-12.

36. HPS2-THRIVE Collaborative Group, Landray MJ, Haynes R, Hopewell JC, Parish S, Aung T, et al. Effects of extended-release niacin with laropiprant in high-risk patients. N Engl J Med. 2014;371(3):203-12.

37. D'Amelio M, Terruso V, Famoso G, Di Benedetto N, Realmuto S, Valentino F, et al. Early and late mortality of spontaneous hemorrhagic transformation of ischemic stroke. J Stroke Cerebrovasc Dis. 2014;23(4):649-54. 